\title{
Global Exponential Stability of a Unique Almost Periodic Solution for Neutral-Type Cohen-Grossberg Neural Networks with Time Delays
}

\author{
Yongzhi Liao, ${ }^{1}$ Wenquan $\mathrm{Wu}^{2}$ and Tianwei $\mathrm{Zhang}^{3}$ \\ ${ }^{1}$ School of Mathematics and Computer Science, Panzhihua University, Panzhihua, Sichuan 617000, China \\ ${ }^{2}$ Department of Mathematics, ABa Teacher's College, Wenchuan, Sichuan 623002, China \\ ${ }^{3}$ City College, Kunming University of Science and Technology, Kunming 650051, China \\ Correspondence should be addressed to Tianwei Zhang; zhang@kmust.edu.cn
}

Received 11 July 2013; Accepted 23 August 2013

Academic Editor: Yong-Kui Chang

Copyright (C) 2013 Yongzhi Liao et al. This is an open access article distributed under the Creative Commons Attribution License, which permits unrestricted use, distribution, and reproduction in any medium, provided the original work is properly cited.

\begin{abstract}
This paper is concerned with the problem of the existence, uniqueness, and global exponential stability of almost periodic solution for neutral-type Cohen-Grossberg neural networks with time delays. Based on fixed point theory and Lyapunov functional, several sufficient conditions are established for the existence, uniqueness, and global exponential stability of almost periodic solution for the above system. Finally, an example and numerical simulations are given to illustrate the feasibility and effectiveness of our main results.
\end{abstract}

\section{Introduction}

In recent years, the Cohen-Grossberg neural networks [1] have been extensively studied because of their immense potentials of application perspective in different areas such as pattern recognition, optimization, and signal and image processing. It is well known that studies on Cohen-Grossberg neural networks not only involve a discussion of stability properties, but also involve many dynamic behaviors such as periodic oscillatory behavior and almost periodic oscillatory properties. In applications, if the various constituent components of the temporally nonuniform environment is with incommensurable (nonintegral multiples) periods, then one has to consider the environment to be almost periodic since there is no a priori reason to expect the existence of periodic solutions. Therefore, if we consider the effects of the environmental factors, almost periodicity is sometimes more realistic and more general than periodicity. In addition, the (almost) periodic oscillatory behavior is of great interest; it has been found that applications in learning theory [2], which is motivated by the fact that learning usually requires repetition. And in pattern recognition or associative memory, the existence of stable almost periodic encoded patterns (including equilibria or periodic orbits) is an important feature $[3,4]$. Hence, it is of prime importance to study the existence and stability of (almost) periodic solutions for Cohen-Grossberg neural networks with delays. We refer the reader to [5-18] and references therein.

On the other hand, owing to the complicated dynamic properties of the neural cells in the real world, the existing neural network models in many cases can not characterize the properties of a neural reaction process precisely. It is natural and important that systems will contain some information about the derivative of the past state to further describe and model the dynamics for such complex neural reactions. This new type of neural networks is called neutral neural networks or neural networks of neutral type. The motivation for us to study neural networks of neutral type comes from three aspects. First, based on biochemistry experiments, neural information may transfer across chemical reactivity, which results in a neutral-type process. Second, in view of electronics, it has been shown that neutral phenomena exist in large-scale integrated (LSI) circuits. Last, the key point is that cerebra can be considered as a super LSI circuit with chemical reactivity, which reasonably implies that the neutral dynamic behaviors should be included in neural dynamic 
systems [19]. To the best of our knowledge, the problem of global exponential stability of almost periodic solution for neutral-type Cohen-Grossberg neural networks (see [2024]) has not been fully investigated in the literature.

In this papers we consider the following neutral-type Cohen-Grossberg neural networks with time delays:

$$
\begin{aligned}
\dot{u}_{i}(t)= & -a_{i}\left(u_{i}(t)\right) \\
\times & {\left[b_{i}\left(t, u_{i}(t)\right)-\sum_{j=1}^{n} c_{i j}(t) f_{j}\left(u_{j}(t)\right)\right.} \\
& -\sum_{j=1}^{n} d_{i j}(t) g_{j}\left(u_{j}\left(t-\mu_{j}(t)\right)\right) \\
& \left.\quad-\sum_{j=1}^{n} v_{i j}(t) k_{j}\left(\dot{u}_{j}\left(t-v_{j}(t)\right)\right)-I_{i}(t)\right],
\end{aligned}
$$

where $x_{i}$ denotes the state variable associated to the $i$ th neuron, $a_{i}$ represents an amplification function, $b_{i}$ is an appropriately behaved function; $f_{j}, g_{j}$, and $k_{j}$ denote the normal and the delayed activation function; $c_{i j}, d_{i j}$, and $v_{i j}$ denote the connection strengths of the $j$ th neuron on the $i$ th neuron at time $t$, respectively; $\mu_{j} \geq 0$ and $\nu_{j} \geq 0$ are the delays caused during the switching and transmission processes; $I_{i}$ denotes external input to the $i$ th neuron at time $t, i, j=$ $1,2, \ldots, n$.

Let $C(\mathbb{X}, \mathbb{Y})$ and $C^{1}(\mathbb{X}, \mathbb{Y})$ be the space of continuous functions and continuously differential functions which map $\mathbb{X}$ into $\mathbb{Y}$, respectively. In particular, $C(\mathbb{X}):=C(\mathbb{X}, \mathbb{X})$, $C^{1}(\mathbb{X}):=C^{1}(\mathbb{X}, \mathbb{X})$. For any bounded function, $f \in C(\mathbb{R})$, $f^{+}=\sup _{s \in \mathbb{R}}|f(s)|, f^{-}=\inf _{s \in \mathbb{R}}|f(s)|$.

Throughout this paper, we set

$$
x=\left\{x_{i}\right\}=\left(x_{1}, x_{2}, \ldots, x_{n}\right) \in \mathbb{R}^{n} .
$$

For $x=\left\{x_{i}\right\} \in \mathbb{R}^{n}$, we define the norm $\|x\|=$ $\max _{1 \leq i \leq n}\left|x_{i}\right|$.

We list some assumptions which will be used in this paper.

$\left(H_{1}\right) c_{i j}, d_{i j}, v_{i j}, \mu_{i j}, v_{i j}$, and $I_{i j}$ are continuous almost periodic functions, $i, j=1,2, \ldots, n$.

$\left(H_{2}\right) a_{i} \in C(\mathbb{R})$ and there exist positive constants $\underline{a}_{i}$ and $\bar{a}_{i}$ such that $0<\underline{a}_{i} \leq a_{i}(t) \leq \bar{a}_{i}$, for all $t \in \mathbb{R}, i=$ $1,2, \ldots, n$

$\left(H_{3}\right) b_{i} \in C\left(\mathbb{R}^{2}, \mathbb{R}\right)$ is almost periodic about the first argument, and there are positive constants $p_{i}$ and $q_{i}$ such that $p_{i} \leq\left(\partial b_{i}(t, u) / \partial u\right) \leq q_{i}$ and $b_{i}(t, 0)=0$, for all $(t, u) \in(\mathbb{R}, \mathbb{R}), i=1,2, \ldots, n$.
$\left(H_{4}\right)$ There exist positive constants $L_{j}^{f}, L_{j}^{g}, L_{j}^{k}$, and $L_{j}^{a}$ such that

$$
\begin{aligned}
& \left|f_{j}(u)-f_{j}(v)\right| \leq L_{j}^{f}|u-v|, \\
& \left|g_{j}(u)-g_{j}(v)\right| \leq L_{j}^{g}|u-v|, \\
& \left|k_{j}(u)-k_{j}(v)\right| \leq L_{j}^{k}|u-v|, \\
& \left|a_{j}(u)-a_{j}(v)\right| \leq L_{j}^{a}|u-v|,
\end{aligned}
$$

for all $u, v \in \mathbb{R}, j=1,2, \ldots, n$.

The organization of this paper is as follows. In Section 2, we give some basic definitions and necessary lemmas which will be used in later sections. In Sections 3 and 4, by using a fixed point theorem and constructing suitable Lyapunov functional, we obtain some sufficient conditions ensuring the existence, uniqueness, and global exponential stability of almost periodic solution of system (1). Finally, an example and numerical simulations are given to illustrate that our results are feasible.

\section{Preliminaries}

First of all, we shall transform system (1) and state some notations, which will be used in later sections.

From $\left(H_{2}\right)$, the antiderivative of $1 / a_{i}\left(u_{i}\right)$ exists. We choose an antiderivative $h_{i}\left(u_{i}\right)$ of $1 / a_{i}\left(u_{i}\right)$ that satisfies $h_{i}(0)=0$. Obviously, $\left(\mathrm{d} / \mathrm{d} u_{i}\right) h_{i}\left(u_{i}\right)=1 / a_{i}\left(u_{i}\right)$. By $a_{i}\left(u_{i}\right)>0$, we obtain that $h_{i}\left(u_{i}\right)$ is strictly monotone increasing about $u_{i}$. In view of derivative theorem for inverse function, the inverse function $h_{i}^{-1}\left(u_{i}\right)$ of $h_{i}\left(u_{i}\right)$ is differential and $\left(\mathrm{d} / \mathrm{d} u_{i}\right) h_{i}^{-1}\left(u_{i}\right)=a_{i}\left(u_{i}\right)$. By $\left(H_{3}\right)$, composition function $b_{i}\left(t, h_{i}^{-1}(z)\right)$ is differentiable. Denote $x_{i}=h_{i}\left(u_{i}\right)$. It is easy to see that $\dot{x}_{i}(t)=\dot{u}_{i}(t) / a_{i}\left(u_{i}\right)$, $u_{i}(t)=h_{i}^{-1}\left(x_{i}(t)\right)$, and $\dot{u}_{i}(t)=a_{i}\left(x_{i}(t)\right) \dot{x}_{i}(t)$. Substituting these equalities into system (1), we get

$$
\begin{aligned}
\dot{x}_{i}(t)= & -b_{i}\left(t, h_{i}^{-1}\left(x_{i}(t)\right)\right) \\
& +\sum_{j=1}^{n} c_{i j}(t) f_{j}\left(h_{j}^{-1}\left(x_{j}(t)\right)\right) \\
+ & \sum_{j=1}^{n} d_{i j}(t) g_{j}\left(h_{j}^{-1}\left(x_{j}\left(t-\mu_{j}(t)\right)\right)\right) \\
+ & \sum_{j=1}^{n} v_{i j}(t) k_{j}\left(a_{j}\left(x_{j}\left(t-v_{j}(t)\right)\right)\right. \\
& \left.\times \dot{x}_{j}\left(t-v_{j}(t)\right)\right)+I_{i}(t),
\end{aligned}
$$


which can be rewritten as

$$
\begin{aligned}
\dot{x}_{i}(t)= & -w_{i}\left(t, x_{i}(t)\right) x_{i}(t) \\
& +\sum_{j=1}^{n} c_{i j}(t) f_{j}\left(h_{j}^{-1}\left(x_{j}(t)\right)\right) \\
& +\sum_{j=1}^{n} d_{i j}(t) g_{j}\left(h_{j}^{-1}\left(x_{j}\left(t-\mu_{j}(t)\right)\right)\right) \\
& +\sum_{j=1}^{n} v_{i j}(t) k_{j}\left(a_{j}\left(x_{j}\left(t-v_{j}(t)\right)\right)\right. \\
& \left.\quad \times \dot{x}_{j}\left(t-v_{j}(t)\right)\right)+I_{i}(t),
\end{aligned}
$$

where $w_{i}\left(t, x_{i}(t)\right)=\left.\left(\partial b_{i}\left(t, h_{i}^{-1}(z)\right) / \partial z\right)\right|_{z=\xi_{i}}, \xi_{i}$ is between 0 and $x_{i}, i=1,2, \ldots, n$.

By $\left(H_{2}\right),\left(H_{3}\right)$, and the definition of $h_{i}^{-1}$, we obtain that $b_{i}\left(t, h_{i}^{-1}\left(x_{i}\right)\right)$ is strictly monotone increasing about $x_{i}$. Hence, $w_{i}\left(t, x_{i}\right)$ is unique for any $x_{i}$ and continuous about $x_{i}$; moreover,

$$
\alpha_{i}=\underline{a}_{i} p_{i} \leq w_{i}\left(t, x_{i}\right) \leq \bar{a}_{i} q_{i}=\beta_{i}, \quad i=1,2, \ldots, n .
$$

From the definition of $h_{i}^{-1}$, using Lagrange mean-value theorem, one gets

$$
\left|h_{i}^{-1}(u)-h_{i}^{-1}(v)\right| \leq \bar{a}_{i}|u-v|, \quad \forall u, v \in \mathbb{R}, i=1,2, \ldots, n .
$$

The existence and global exponential stability of almost periodic solution for system (1) are equivalent to the existence and global exponential stability of almost periodic solution for system (5), respectively. So, we investigate the existence and global exponential stability of almost periodic solution for system (5).

Let $\tau:=\max _{1 \leq i \leq n}\left\{\mu_{i}^{+}, v_{i}^{+}\right\}$. The initial conditions associated with system (5) are of the form

$$
\begin{gathered}
x_{i}(s)=\varphi_{i}(s), \quad \dot{x}_{i}(s)=\dot{\varphi}_{i}(s), \\
\forall s \in[-\tau, 0], \quad \varphi_{i} \in C^{1}([-\tau, 0], \mathbb{R}), \quad i=1,2, \ldots, n .
\end{gathered}
$$

Now, let us state the following definitions and lemmas, which will be useful in proving our main result.

Definition 1 (see [25]). $x \in C\left(\mathbb{R}, \mathbb{R}^{n}\right)$ is called almost periodic, if for any $\epsilon>0$, it is possible to find a real number $l=l(\epsilon)>0$, for any interval with length $l(\epsilon)$, there exists a number $\tau=\tau(\epsilon)$ in this interval such that $\|x(t+\tau)-x(t)\|<\epsilon$, for all $t \in \mathbb{R}$. The collection of those functions is denoted by $A P\left(\mathbb{R}, \mathbb{R}^{n}\right)$.

Definition 2 (see [25]). Let $y \in C\left(\mathbb{R}, \mathbb{R}^{n}\right)$ and $P(t)$ be an $n \times n$ continuous matrix defined on $\mathbb{R}$. The linear system

$$
\dot{y}(t)=P(t) y(t)
$$

is said to be an exponential dichotomy on $\mathbb{R}$ if there exist constants $k, \lambda>0$, projection $S$, and the fundamental matrix $Y(t)$ satisfying

$$
\begin{gathered}
\left\|Y(t) S Y^{-1}(s)\right\| \leq k e^{-\lambda(t-s)}, \quad \forall t \geq s, \\
\left\|Y(t)(I-S) Y^{-1}(s)\right\| \leq k e^{-\lambda(s-t)}, \quad \forall t \leq s .
\end{gathered}
$$

Lemma 3 (see [25]). If the linear system $\dot{y}(t)=P(t) y(t)$ has an exponential dichotomy, then almost periodic system

$$
\dot{y}(t)=P(t) y(t)+g(t)
$$

has a unique almost periodic solution $y(t)$ which can be expressed as follows:

$$
\begin{aligned}
y(t)= & \int_{-\infty}^{t} Y(t) S Y^{-1}(s) g(s) d s \\
& -\int_{t}^{\infty} Y(t)(I-S) Y^{-1}(s) g(s) d s .
\end{aligned}
$$

Lemma 4 (see [25]). Let $a_{i}(t)$ be an almost periodic function and

$$
M\left[a_{i}\right]=\lim _{T \rightarrow \infty} \frac{1}{T} \int_{t}^{t+T} a_{i}(s) d s>0, \quad i=1,2, \ldots, n .
$$

Then the linear system $\dot{y}(t)=-A(t) y(t)$ admits an exponential dichotomy, where $A(t)=\operatorname{diag}\left\{a_{1}(t), a_{2}(t), \ldots, a_{n}(t)\right\}$.

Definition 5. The almost periodic solution $x=\left\{x_{i}\right\}$ of system (5) with the initial value $\varphi=\left\{\varphi_{i}\right\}$ is said to be globally exponentially stable if there exist constants $\omega>0$ and $M \geq 1$, for any solution $y=\left\{y_{i}\right\}$ of system (5) with initial value $\phi=\left\{\phi_{i}\right\}$ such that

$$
\|x-y\| \leq M\|\varphi-\phi\|_{\tau} e^{-\omega t}, \quad \forall t>0,
$$

where

$$
\|\varphi-\phi\|_{\tau}:=\sum_{i=1}^{n} \sup _{s \in[-\tau, 0]}\left\{\left|\varphi_{i}(s)-\phi_{i}(s)\right|+\left|\dot{\varphi}_{i}(s)-\dot{\phi}_{i}(s)\right|\right\} .
$$

Lemma 6 (see $[26,27])$. Let $\left(\mathbb{X},\|\cdot\|_{\mathbb{X}}\right)$ be a Banach space. Assume that $0 \in \Omega$ is an open bounded subset of $\mathbb{X}$ and $T: \bar{\Omega} \rightarrow \mathbb{X}$ is completely continuous satisfying

$$
\|T x\|_{\mathbb{X}} \leq\|x\|_{\mathbb{X}}, \quad \forall x \in \partial \Omega,
$$

then $T$ has a fixed point in $\bar{\Omega}$.

$$
\begin{aligned}
& \text { Let } \\
& \mathbb{X}=\left\{x \in A P\left(\mathbb{R}, \mathbb{R}^{n}\right) \cap C^{1}\left(\mathbb{R}, \mathbb{R}^{n}\right): \dot{x} \in A P\left(\mathbb{R}, \mathbb{R}^{n}\right)\right\}
\end{aligned}
$$

with the norm $\|x\|_{\mathbb{X}}=\max \left\{\sup _{s \in \mathbb{R}}\|x(s)\|, \sup _{s \in \mathbb{R}}\|\dot{x}(s)\|\right\}$. Then $\mathbb{X}$ is a Banach space with the norm $\|\cdot\|_{\mathbb{X}}$.

For given positive constant $\gamma$, define an open bounded subset $\Omega_{\gamma}$ in $\mathbb{X}$ by

$$
\Omega=\left\{x \in \mathbb{X}:\|x\|_{\mathbb{X}}<\gamma\right\} .
$$


By Lemmas 3 and 4, system (5) has a unique almost periodic solution

$$
x^{\varphi}=\left\{x_{i}^{\varphi_{i}}\right\}=\left(x_{1}^{\varphi_{1}}, x_{2}^{\varphi_{2}}, \ldots, x_{n}^{\varphi_{n}}\right)^{T}
$$

which can be expressed as follows:

$$
x_{i}^{\varphi_{i}}=\int_{-\infty}^{t} e^{-\int_{s}^{t} w_{i}\left(r, \varphi_{i}(r)\right) \mathrm{d} r} F_{i}(s, \varphi(s)) \mathrm{d} s,
$$

where

$$
\begin{aligned}
F_{i}(t, \varphi(t))= & \sum_{j=1}^{n} c_{i j}(t) f_{j}\left(h_{j}^{-1}\left(\varphi_{j}(t)\right)\right) \\
+ & +\sum_{j=1}^{n} d_{i j}(t) g_{j}\left(h_{j}^{-1}\left(\varphi_{j}\left(t-\mu_{j}(t)\right)\right)\right) \\
+ & \sum_{j=1}^{n} v_{i j}(t) k_{j}\left(a_{j}\left(\varphi_{j}\left(t-v_{j}(t)\right)\right)\right. \\
& \left.\times \dot{\varphi}_{j}\left(t-v_{j}(t)\right)\right)+I_{i}(t),
\end{aligned}
$$

where $i=1,2, \ldots, n$, for all $t \in \mathbb{R}$.

Let the map $T: \mathbb{X} \rightarrow \mathbb{X}$ be defined by

$$
T(\varphi)=\left\{T_{i}(\varphi)\right\}=\left\{x_{i}^{\varphi_{i}}\right\}, \quad \forall \varphi=\left\{\varphi_{i}\right\} \in \mathbb{X} .
$$

Lemma 7. $T: \bar{\Omega}_{\gamma} \rightarrow \mathbb{X}$ is completely continuous.

Proof. Obviously, $T: \bar{\Omega} \rightarrow \mathbb{X}$ is continuous. Next, we show that $T$ maps bounded set into itself. Assume $c$ is a positive constant and $\varphi \in \bar{\Omega}_{c}=\left\{x \in \Omega:\|x\|_{\mathbb{X}} \leq c\right\}$. By the almost periodicity of system (1), there exists a constant $K>0$ such that

$$
\max _{1 \leq i \leq n} \sup _{s \in \mathbb{R}, \varphi \in \bar{\Omega}_{c}}\left\{F_{i}(s, 0), F_{i}(s, \varphi)\right\}<K .
$$

Assume that $\varphi \in \bar{\Omega}_{\gamma}$, then

$$
\begin{aligned}
& \left|F_{i}(t, \varphi(t))-F_{i}(t, 0)\right| \\
& \leq\left|\sum_{j=1}^{n} c_{i j}(t) f_{j}\left(h_{j}^{-1}\left(\varphi_{j}(t)\right)\right)-\sum_{j=1}^{n} c_{i j}(t) f_{j}\left(h_{j}^{-1}(0)\right)\right| \\
& +\mid \sum_{j=1}^{n} d_{i j}(t) g_{j}\left(h_{j}^{-1}\left(\varphi_{j}\left(t-\mu_{j}(t)\right)\right)\right) \\
& \quad-\sum_{j=1}^{n} d_{i j}(t) g_{j}\left(h_{j}^{-1}(0)\right) \mid
\end{aligned}
$$

$$
\begin{aligned}
& +\mid \sum_{j=1}^{n} v_{i j}(t) k_{j}\left(a_{j}\left(\varphi_{j}\left(t-v_{j}(t)\right)\right) \dot{\varphi}_{j}\left(t-v_{j}(t)\right)\right) \\
& \quad-\sum_{j=1}^{n} v_{i j}(t) k_{j}(0) \mid
\end{aligned}
$$$$
\leq \sum_{j=1}^{n}\left[c_{i j}^{+} L_{j}^{f} \bar{a}_{j}+d_{i j}^{+} L_{j}^{g} \bar{a}_{j}+v_{i j}^{+} M_{j}^{k}\left(\bar{a}_{j}+L_{j}^{a} \gamma\right)\right]\|\varphi\|_{\mathbb{X}},
$$

which implies that

$$
\begin{aligned}
&\left|T_{i}(\varphi)\right|= \int_{-\infty}^{t} e^{-\int_{s}^{t} w_{i}\left(r, \varphi_{i}(r)\right) \mathrm{d} r}\left|F_{i}(s, \varphi(s))-F_{i}(s, 0)\right| \mathrm{d} s \\
&+\int_{-\infty}^{t} e^{-\int_{s}^{t} w_{i}\left(r, \varphi_{i}(r)\right) \mathrm{d} r}\left|F_{i}(s, 0)\right| \mathrm{d} s \\
& \leq \frac{1}{\alpha_{i}} \sum_{j=1}^{n}\left[c_{i j}^{+} L_{j}^{f} \bar{a}_{j}+d_{i j}^{+} L_{j}^{g} \bar{a}_{j}+v_{i j}^{+} M_{j}^{k}\left(\bar{a}_{j}+L_{j}^{a} \gamma\right)\right] \\
& \times\|\varphi\|_{X}+\frac{K}{\alpha_{i}} \\
& \leq \frac{1}{\alpha_{i}} \sum_{j=1}^{n}\left[c_{i j}^{+} L_{j}^{f} \bar{a}_{j}+d_{i j}^{+} L_{j}^{g} \bar{a}_{j}+v_{i j}^{+} M_{j}^{k}\left(\bar{a}_{j}+L_{j}^{a} \gamma\right)\right] \\
& \times c+\frac{K}{\alpha_{i}}, \\
&\left|\dot{T}_{i}(\varphi)\right|=\left|-w_{i}\left(t, \varphi_{i}(t)\right) T_{i}(\varphi)+F_{i}(t, \varphi)\right| \\
& \leq \beta_{i}\left\{\frac { 1 } { \alpha _ { i } } \sum _ { j = 1 } ^ { n } \left[c_{i j}^{+} L_{j}^{f} \bar{a}_{j}+d_{i j}^{+} L_{j}^{g} \bar{a}_{j}\right.\right. \\
&\left.\left.+v_{i j}^{+} M_{j}^{k}\left(\bar{a}_{j}+L_{j}^{a} \gamma\right)\right] c+\frac{K}{\alpha_{i}}\right\}+K,
\end{aligned}
$$

where $i=1,2, \ldots, n$. Therefore, $T \bar{\Omega}_{c}$ is a family of uniformly bounded and equicontinuous subsets. Using Arzela-Ascoli theorem, we obtain that $T \bar{\Omega}_{c}$ is relatively compact. Hence, $T$ is completely continuous. The proof of this lemma is complete.

\section{Existence of Almost Periodic Solution}

In this section, we study the existence of almost periodic solutions of system (1). 
Theorem 8. Assume that $\left(H_{1}\right)-\left(H_{4}\right)$ hold, suppose further that

$$
\begin{gathered}
\left(H_{5}\right) \theta=\max \left\{\theta_{1}, \theta_{2}\right\}<1, \text { where } \\
\theta_{1}=\max _{1 \leq i \leq n}\left\{\frac{1}{\alpha_{i}} \sum_{j=1}^{n}\left(c_{i j}^{+} L_{j}^{f} \bar{a}_{j}+d_{i j}^{+} L_{j}^{g} \bar{a}_{j}+v_{i j}^{+} L_{j}^{k} \bar{a}_{j}\right)\right\}, \\
\theta_{2}=\max _{1 \leq i \leq n}\left\{\left[1+\frac{\beta_{i}}{\alpha_{i}}\right] \sum_{j=1}^{n}\left(c_{i j}^{+} L_{j}^{f} \bar{a}_{j}+d_{i j}^{+} L_{j}^{g} \bar{a}_{j}+v_{i j}^{+} L_{j}^{k} \bar{a}_{j}\right)\right\},
\end{gathered}
$$

then system (1) admits at least one almost periodic solution.

Proof. Define

$$
\Omega_{r_{0}}=\left\{x \in \mathbb{X}:\|x\|_{\mathbb{X}}<r_{0}=\frac{\delta}{1-\theta}\right\},
$$

where $\delta=\max \left\{\delta_{1}, \delta_{2}\right\}$,

$$
\begin{array}{r}
\delta_{1}=\max _{1 \leq i \leq n}\left\{\frac{1}{\alpha_{i}} \sum_{j=1}^{n}\left(c_{i j}^{+} f_{j}(0)+d_{i j}^{+} g_{j}(0)+v_{i j}^{+} k_{j}(0)\right)+\frac{I_{i}^{+}}{\alpha_{i}}\right\}, \\
\delta_{2}=\max _{1 \leq i \leq n}\left\{[ 1 + \frac { \beta _ { i } } { \alpha _ { i } } ] \left[\sum _ { j = 1 } ^ { n } \left(c_{i j}^{+} f_{j}(0)+d_{i j}^{+} g_{j}(0)\right.\right.\right. \\
\left.\left.\left.+v_{i j}^{+} k_{j}(0)\right)+I_{i}^{+}\right]\right\} .
\end{array}
$$

Consider the following nonlinear operator:

$$
T(\phi)=\left\{T_{i}(\phi)\right\}=\left\{x_{i}^{\phi_{i}}\right\}, \quad \forall \phi=\left\{\phi_{i}\right\} \in \bar{\Omega}_{r_{0}} .
$$

For $\forall \varphi=\left\{\varphi_{\mathrm{i}}\right\} \in \partial \Omega_{\mathrm{r}_{0}}$, we have $\|\varphi\|_{\mathbb{X}}=r_{0}$. So, we obtain

$$
\begin{aligned}
\|T(\varphi(t))\|=\max _{1 \leq i \leq n}\left|T_{i}(\varphi(t))\right| & \\
=\max _{1 \leq i \leq n} \mid \int_{-\infty}^{t} e^{-\int_{s}^{t} w_{i}\left(r, \varphi_{i}(r)\right) \mathrm{d} r} & \\
\times & {\left[\sum_{j=1}^{n} c_{i j}(s) f_{j}\left(h_{j}^{-1}\left(\varphi_{j}(s)\right)\right)\right.} \\
& +\sum_{j=1}^{n} d_{i j}(s) g_{j} \\
& \times\left(h_{j}^{-1}\left(\varphi_{j}\left(s-\mu_{j}(s)\right)\right)\right) \\
& +\sum_{j=1}^{n} v_{i j}(s) k_{j} \\
& \times\left(a_{j}\left(\varphi_{j}\left(s-v_{j}(s)\right)\right)\right. \\
& \left.\left.\times \dot{\varphi}_{j}\left(s-v_{j}(s)\right)\right)+I_{i}(s)\right] \mathrm{d} s \mid
\end{aligned}
$$

$$
\begin{aligned}
\leq \max _{1 \leq i \leq n}\left\{\int_{-\infty}^{t} e^{-\int_{s}^{t} w_{i}\left(r, \varphi_{i}(r)\right) \mathrm{d} r}\right. \\
\quad \times\left[\sum _ { j = 1 } ^ { n } \left(c_{i j}^{+} L_{j}^{f} \bar{a}_{j}+d_{i j}^{+} L_{j}^{g} \bar{a}_{j}\right.\right. \\
\left.+v_{i j}^{+} L_{j}^{k} \bar{a}_{j}\right)\|\varphi\|_{\mathbb{X}} \\
+\sum_{j=1}^{n}\left(c_{i j}^{+} f_{j}(0)+d_{i j}^{+} g_{j}(0)\right.
\end{aligned}
$$$$
\left.\left.\left.+v_{i j}^{+} k_{j}(0)\right)+I_{i}^{+}\right] \mathrm{d} s\right\}
$$$$
\leq \max _{1 \leq i \leq n}\left\{\frac{1}{\alpha_{i}} \sum_{j=1}^{n}\left(c_{i j}^{+} L_{j}^{f} \bar{a}_{j}+d_{i j}^{+} L_{j}^{g} \bar{a}_{j}+v_{i j}^{+} L_{j}^{k} \bar{a}_{j}\right)\right\} r_{0}
$$$$
+\max _{1 \leq i \leq n}\left\{\frac { 1 } { \alpha _ { i } } \sum _ { j = 1 } ^ { n } \left(c_{i j}^{+} f_{j}(0)+d_{i j}^{+} g_{j}(0)\right.\right.
$$$$
\left.\left.+v_{i j}^{+} k_{j}(0)\right)+\frac{I_{i}^{+}}{\alpha_{i}}\right\}
$$$$
:=\theta_{1} r_{0}+\delta_{1},
$$$$
\|\dot{T}(\varphi(t))\|=\max _{1 \leq i \leq n}\left|\dot{T}_{i}(\varphi(t))\right|
$$

$$
=\max _{1 \leq i \leq n}\left|-w_{i}(t, \varphi) T_{i}(\varphi)+F_{i}(t, \varphi(t))\right|
$$$$
\leq \max _{1 \leq i \leq n}\left\{\left[1+\frac{\beta_{i}}{\alpha_{i}}\right]\right.
$$$$
\times \sum_{j=1}^{n}\left(c_{i j}^{+} L_{j}^{f} \bar{a}_{j}+d_{i j}^{+} L_{j}^{g} \bar{a}_{j}\right.
$$$$
\left.\left.+v_{i j}^{+} L_{j}^{k} \bar{a}_{j}\right)\right\} r_{0}
$$

$$
+\max _{1 \leq i \leq n}\left\{\left[1+\frac{\beta_{i}}{\alpha_{i}}\right]\right.
$$$$
\times\left[\sum _ { j = 1 } ^ { n } \left(c_{i j}^{+} f_{j}(0)+d_{i j}^{+} g_{j}(0)\right.\right.
$$$$
\left.\left.\left.+v_{i j}^{+} k_{j}(0)\right)+I_{i}^{+}\right]\right\}
$$

$$
:=\theta_{2} r_{0}+\delta_{2}
$$


which yield

$$
\begin{aligned}
\|T(\varphi)\|_{\mathbb{X}} & =\max \left\{\sup _{s \in \mathbb{R}}\|T(\varphi(s))\|, \sup _{s \in \mathbb{R}}\|\dot{T}(\varphi(s))\|\right\} \\
& \leq \max \left\{\theta_{1}, \theta_{2}\right\} r_{0}+\max \left\{\delta_{1}, \delta_{2}\right\} \\
& =\theta r_{0}+\delta \\
& \leq \frac{\delta}{1-\theta} \\
& =r_{0} \\
& =\|\varphi\|_{\mathbb{X}}, \quad \forall \varphi \in \partial \Omega_{r_{0}} .
\end{aligned}
$$

By Lemma 6, there exists at least one fixed point $\varphi_{0} \in \bar{\Omega}$ satisfying $T\left(\varphi_{0}\right)=\varphi_{0}$, which implies that system (5) has at least one almost periodic solution $\varphi_{0}$ with $\left\|\varphi_{0}\right\|_{\mathbb{X}} \leq r_{0}$. That is, system (1) has at least one almost periodic solution. This completes the proof.

\section{Global Exponential Stability of Almost Periodic Solution}

Theorem 9. Assume that $\left(H_{1}\right)-\left(H_{5}\right)$ hold, suppose further that

$\left(H_{6}\right)$ there exists a positive constant $\lambda<1$ such that

$$
\begin{aligned}
& \Theta_{1}=\max _{1 \leq i \leq n} \sup _{s \in \mathbb{R}}\left\{-\alpha_{i}+\lambda \beta_{i}+(1+\lambda)\right. \\
& \times \sum_{j=1}^{n}\left|c_{i j}(s)\right| L_{j}^{f} \bar{a}_{j} \\
& +\sum_{j=1}^{n}(1+\lambda)\left|d_{i j}(s)\right| L_{j}^{g} \bar{a}_{j} \\
& \left.+\sum_{j=1}^{n}(1+\lambda)\left|v_{i j}(s)\right| L_{j}^{k} L_{j}^{a} r_{0}\right\}<0, \\
& \Theta_{2}=\max _{1 \leq i, j \leq n} \sup _{s \in \mathbb{R}}\left\{\sum_{j=1}^{n} \lambda\left|v_{i j}(s)\right| L_{j}^{k} \bar{a}_{j}\right. \\
& \left.+\sum_{i=1}^{n}\left|v_{i j}(s)\right| L_{j}^{k} \bar{a}_{j}-\lambda\left(1-\dot{v}_{j}^{+}\right)\right\}<0,
\end{aligned}
$$

where $r_{0}$ is defined as that in Theorem 8.

Then system (1) has a unique almost periodic solution, which is globally exponentially stable.

Proof. It follows from Theorem 8 that system (5) has at least one almost periodic solution $y=\left\{y_{i}\right\}$ with initial value $\phi=\left\{\phi_{i}\right\}$. Next we show that the almost periodic solution $y$ is globally exponentially stable.
Make a transformation for system (5): $x_{i}=z_{i}-y_{i}, i=$ $1,2, \ldots, n$, where $z=\left\{z_{i}\right\}$ is arbitrary solution of system (5) with initial value $\psi=\left\{\psi_{i}\right\}$.

By $\left(H_{6}\right)$, there exists a small enough positive constant $\omega$ such that

$$
\begin{aligned}
\Theta_{1 \omega}=\max _{1 \leq i \leq n} \sup _{s \in \mathbb{R}}\{\omega & \alpha_{i}+\lambda \beta_{i}+(1+\lambda) \\
& \times \sum_{j=1}^{n}\left|c_{i j}(s)\right| L_{j}^{f} \bar{a}_{j} \\
& +\sum_{j=1}^{n}(1+\lambda)\left|d_{i j}(s)\right| L_{j}^{g} \bar{a}_{j} e^{\omega \mu_{j}^{+}} \\
& \left.+\sum_{j=1}^{n}(1+\lambda)\left|v_{i j}(s)\right| L_{j}^{k} L_{j}^{a} r_{0} e^{\omega v_{j}^{+}}\right\}<0,
\end{aligned}
$$

$\Theta_{2 \omega}=\max _{1 \leq i, j \leq n} \sup _{s \in \mathbb{R}}\left\{\sum_{j=1}^{n} \lambda\left|v_{i j}(s)\right| L_{j}^{k} \bar{a}_{j}\right.$

$$
\left.+\sum_{i=1}^{n}\left|v_{i j}(s)\right| L_{j}^{k} \bar{a}_{j}-\lambda e^{-\omega v_{j}^{u}}\left(1-\dot{v}_{j}^{+}\right)\right\}<0 .
$$

Define

$$
V(t)=V_{1}(t)+V_{2}(t)
$$

where

$$
\begin{gathered}
V_{1}(t)=\sum_{i=1}^{n} e^{\omega t}\left|x_{i}(t)\right|, \\
V_{2}(t)=\lambda \sum_{i=1}^{n} \int_{t-v_{i}(t)}^{t} e^{\omega s}\left|\dot{x}_{i}(s)\right| \mathrm{d} s .
\end{gathered}
$$

In view of system (5), we have

$$
\begin{aligned}
& D^{+} V_{1}(t) \leq \omega \sum_{i=1}^{n} e^{\omega t}\left|x_{i}(t)\right| \\
& +\sum_{i=1}^{n} e^{\omega t} \operatorname{sgn}\left(x_{i}(t)\right) \dot{x}_{i}(t) \\
& \leq \omega \sum_{i=1}^{n} e^{\omega t}\left|x_{i}(t)\right| \\
& +\sum_{i=1}^{n} e^{\omega t}\left\{-\left[b_{i}\left(t, h_{i}^{-1}\left(z_{i}(t)\right)\right)\right.\right. \\
& \left.-b_{i}\left(t, h_{i}^{-1}\left(y_{i}(t)\right)\right)\right] \\
& \times \operatorname{sgn}\left(z_{i}(t)-y_{i}(t)\right)
\end{aligned}
$$




$$
\begin{aligned}
+ & \mid \sum_{j=1}^{n} c_{i j}(t) f_{j}\left(h_{j}^{-1}\left(z_{j}(t)\right)\right) \\
& -\sum_{j=1}^{n} c_{i j}(t) f_{j}\left(h_{j}^{-1}\left(y_{j}(t)\right)\right) \mid \\
+ & \mid \sum_{j=1}^{n} d_{i j}(t) g_{j}\left(h_{j}^{-1}\left(z_{j}\left(t-\mu_{j}(t)\right)\right)\right) \\
& -\sum_{j=1}^{n} d_{i j}(t) g_{j}\left(h_{j}^{-1}\left(y_{j}\left(t-\mu_{j}(t)\right)\right)\right) \mid \\
+ & \mid \sum_{j=1}^{n} v_{i j}(t) k_{j} \\
& \times\left(a_{j}\left(z_{j}\left(t-v_{j}(t)\right)\right) \dot{z}_{j}\left(t-v_{j}(t)\right)\right) \\
& +\sum_{j=1}^{n} v_{i j}(t) k_{j} \\
+\sum_{i=1}^{n} e^{\omega t}\left\{\begin{array}{l}
\left.v_{i j}(t)\left|L_{j}^{k} \bar{a}_{j}\right| \dot{x}_{j}\left(t-v_{j}(t)\right) \mid\right\} \\
+\sum_{i=1} e^{\omega t}\left|x_{i}(t)\right|
\end{array}\right. & \times\left(a_{i}(t) \mid\right. \\
& \left.+\sum_{j=1}^{n}\left|c_{i j}(t)\right| L_{j}^{f} \bar{a}_{j} \mid x_{j}\left(t-v_{j}(t) \mid d_{i j}(t)\right)\right) \\
& \left.\left.\times \dot{y}_{j}\left(t-v_{j}^{k} L_{j}^{a} r_{0} \mid x_{j}(t)\right)\right) \mid\right\}
\end{aligned}
$$

Further, it follows from (36) that

$$
\begin{aligned}
D^{+} V_{2}(t) \leq & \lambda \sum_{i=1}^{n} e^{\omega t}\left|\dot{x}_{i}(t)\right|-\lambda \\
& \times \sum_{i=1}^{n} e^{\omega\left(t-v_{i}(t)\right)}\left(1-\dot{v}_{i}(t)\right)\left|\dot{x}_{i}\left(t-v_{i}(t)\right)\right|
\end{aligned}
$$

$$
\begin{aligned}
& \leq \lambda \sum_{i=1}^{n} e^{\omega t}[ \beta_{i}\left|x_{i}(t)\right| \\
&+\sum_{j=1}^{n}\left|c_{i j}(t)\right| L_{j}^{f} \bar{a}_{j}\left|x_{j}(t)\right| \\
&+\sum_{j=1}^{n}\left|d_{i j}(t)\right| L_{j}^{g} \bar{a}_{j}\left|x_{j}\left(t-\mu_{j}(t)\right)\right| \\
&\left.+\sum_{j=1}^{n}\left|v_{i j}(t)\right| L_{j}^{k} L_{j}^{a} r_{0}\left|x_{j}\left(t-v_{j}(t)\right)\right|\right] \\
&-\lambda \sum_{j=1}^{n} e^{\omega t} {\left[e^{-\omega \nu_{j}^{u}}\left(1-\dot{v}_{j}^{+}\right)-\sum_{j=1}^{n}\left|v_{i j}(t)\right| L_{j}^{k} \bar{a}_{j}\right] } \\
& \times\left|\dot{x}_{j}\left(t-v_{j}(t)\right)\right| .
\end{aligned}
$$

By (36) and (37), we have

$$
\begin{aligned}
& D^{+} V(t) \leq \sum_{i=1}^{n}\left[\omega-\alpha_{i}+\lambda \beta_{i}+(1+\lambda)\right. \\
&\left.\quad \times \sum_{j=1}^{n}\left|c_{i j}(t)\right| L_{j}^{f} \bar{a}_{j}\right] e^{\omega t}\left|x_{i}(t)\right| \\
&+\sum_{i=1}^{n} \sum_{j=1}^{n}(1+\lambda)\left|d_{i j}(t)\right| L_{j}^{g} \bar{a}_{j} e^{\omega \mu_{j}^{+}} e^{\omega\left(t-\mu_{j}(t)\right)} \\
&+\left|x_{j}\left(t-\mu_{j}(t)\right)\right| \\
&+\sum_{i=1}^{n} \sum_{j=1}^{n}(1+\lambda)\left|v_{i j}(t)\right| L_{j}^{\mathrm{k}} L_{j}^{a} r_{0} e^{\omega v_{j}^{+}} e^{\omega\left(t-\mu_{j}(t)\right)} \\
& \times\left|x_{j}\left(t-v_{j}(t)\right)\right| \\
&-\sum_{j=1}^{n} e^{\omega t}\left[\lambda e^{-\omega \nu_{j}^{u}}\left(1-\dot{v}_{j}^{+}\right)\right.
\end{aligned}
$$$$
-\sum_{j=1}^{n} \lambda\left|v_{i j}(t)\right| L_{j}^{k} \bar{a}_{j}
$$$$
\left.-\sum_{i=1}^{n}\left|v_{i j}(t)\right| L_{j}^{k} \bar{a}_{j}\right]\left|\dot{x}_{j}\left(t-v_{j}(t)\right)\right| .
$$ 
For $t \leq 0$, note that

$$
\begin{aligned}
\sum_{i=1}^{n} e^{\omega t}\left|x_{i}(t)\right|= & V_{1}(t) \\
\leq & V(t) \\
= & \sum_{i=1}^{n} e^{\omega t}\left|x_{i}(t)\right| \\
& +\lambda \sum_{i=1}^{n} \int_{t-v_{i}(t)}^{t} e^{\omega s}\left|\dot{x}_{i}(s)\right| \mathrm{d} s \\
\leq & \left(1+e^{\omega \tau}\right)\|\psi-\phi\|_{\tau} \\
:= & M\|\psi-\phi\|_{\tau},
\end{aligned}
$$

where $M=1+e^{\omega \tau}$. Next, we claim that

$$
V(t) \leq M\|\psi-\phi\|_{\tau}, \quad \forall t \in[0,+\infty) .
$$

Contrarily, there is $t_{0} \in[0,+\infty)$ such that

$$
\begin{array}{cl}
V\left(t_{0}\right)=M\|\psi-\phi\|_{\tau}, & D^{+} V\left(t_{0}\right)>0, \\
V(t) \leq M\|\psi-\phi\|_{\tau}, & \forall t \in\left[-\tau, t_{0}\right] .
\end{array}
$$

By (38), that we have from (33)

$$
\begin{aligned}
& 0<D^{+} V\left(t_{0}\right) \\
& \leq \sum_{i=1}^{n}\left[\omega-\alpha_{i}+\lambda \beta_{i}+(1+\lambda)\right. \\
& \quad \times \sum_{j=1}^{n}\left|c_{i j}\left(t_{0}\right)\right| L_{j}^{f} \bar{a}_{j} \\
& +\sum_{j=1}^{n}(1+\lambda)\left|d_{i j}\left(t_{0}\right)\right| L_{j}^{g} \bar{a}_{j} e^{\omega \mu_{j}^{+}} \\
& \left.+\sum_{j=1}^{n}(1+\lambda)\left|v_{i j}\left(t_{0}\right)\right| L_{j}^{k} L_{j}^{a} r_{0} e^{\omega \nu_{j}^{+}}\right] M\|\psi-\phi\|_{\tau} \\
& -\sum_{j=1}^{n} e^{\omega t_{0}}\left[\lambda e^{-\omega \nu_{j}^{u}}\left(1-\dot{v}_{j}^{+}\right)\right. \\
& -\sum_{j=1}^{n} \lambda\left|v_{i j}\left(t_{0}\right)\right| L_{j}^{k} \bar{a}_{j} \\
& \left.-\sum_{i=1}^{n}\left|v_{i j}\left(t_{0}\right)\right| L_{j}^{k} \bar{a}_{j}\right]\left|\dot{x}_{j}\left(t_{0}-v_{j}\left(t_{0}\right)\right)\right|
\end{aligned}
$$

$<0$.

This is a contradiction. So, our claim is valid. Therefore,

$$
\sum_{i=1}^{n}\left|x_{i}(t)\right| \leq M\|\psi-\phi\|_{\tau} e^{-\omega t}, \quad \forall t>0 .
$$

Thus, the almost periodic solution of system (5) is globally exponentially stable. That is, the corresponding almost periodic solution of system (1) is globally exponentially stable.

Without loss of generality, we assume that $y=\left\{x_{i}\right\}$ and $z=\left\{z_{i}\right\}$ are two almost periodic solutions of system (5). Then $x=\left\{x_{i}\right\}=z-y=\left\{z_{i}-y_{i}\right\} \in A P\left(\mathbb{R}, \mathbb{R}^{n}\right)$. Similar to the above argument, (43) is valid. Hence,

$$
\lim _{t \rightarrow \infty} \sum_{i=1}^{n}\left|x_{i}(t)\right|=0,
$$

which implies from $x \in A P\left(\mathbb{R}, \mathbb{R}^{n}\right)$ that $x \equiv 0$. That is, $y=z$. So the almost periodic solution of system (5) is unique. This completes the proof.

\section{An Example and Numerical Simulations}

Example 1. Consider the following neutral-type CohenGrossberg neural networks:

$$
\begin{aligned}
\dot{u}_{i}(t)=-a_{i}\left(u_{i}(t)\right)[ & u_{i}(t)-\sum_{j=1}^{2} c_{i j}(t) f\left(u_{j}(t)\right) \\
& -\sum_{j=1}^{2} d_{i j}(t) g\left(u_{j}\left(t-0.01 \sin ^{2}(\sqrt{2} t)\right)\right) \\
& \left.-\sum_{j=1}^{2} 0.1 k\left(\dot{u}_{j}(t-1)\right)-I_{i}(t)\right],
\end{aligned}
$$

where $i=1,2, f(s)=g(s)=k(s)=0.1 s$,

$$
\begin{aligned}
\left(\begin{array}{l}
a_{1}(s) \\
a_{2}(s)
\end{array}\right) & =\left(\begin{array}{c}
0.11+0.01 \sin (\sqrt{2} s) \\
0.11+0.01 \cos (\sqrt{13} s)
\end{array}\right), \\
\left(\begin{array}{l}
I_{1}(s) \\
I_{2}(s)
\end{array}\right) & =\left(\begin{array}{c}
0.01 \sin (\sqrt{7} s) \\
0.01 \sin (s)
\end{array}\right), \\
\left(\begin{array}{ccc}
c_{11}(s) & c_{12}(s) \\
c_{21}(s) & c_{22}(s)
\end{array}\right) & =\left(\begin{array}{ll}
d_{11}(s) & d_{12}(s) \\
d_{21}(s) & d_{22}(s)
\end{array}\right) \\
& =\left(\begin{array}{cc}
0.1 \sin (\sqrt{2} s) & 0 \\
0.1 \cos (\sqrt{3} s) & 0.1
\end{array}\right), \quad \forall s \in \mathbb{R} .
\end{aligned}
$$

Then system (45) has a unique almost periodic solution, which is globally exponentially stable.

Proof. Corresponding to system (1), $\underline{a}_{i}=\alpha_{i}=0.1, \bar{a}_{i}=\beta_{i}=$ $0.12, c_{i j}^{+}=d_{i j}^{+}=v_{i j}^{+}=0.1, L_{j}^{f}=L_{j}^{g}=L_{j}^{k}=0.1, L_{j}^{a}=0.05, I_{i}^{+}=$ 0.01 , and $i, j=1,2$. Taking $\lambda=0.5$, by an easy calculation, we obtain

$$
\theta=0.072, \quad \delta=0.1, \quad r_{0}=0.1 .
$$

Further, we also have

$$
\Theta_{1} \leq-0.03<0, \quad \Theta_{2} \leq-0.49<0 .
$$




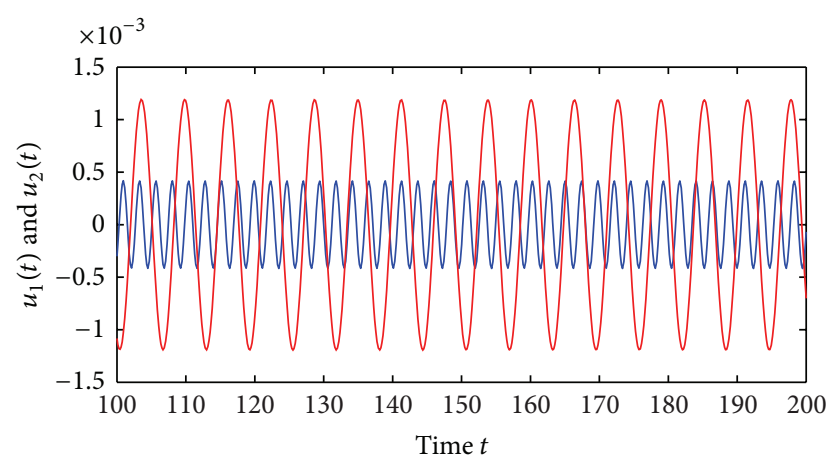

FIgURE 1: State variables $u_{1}$ and $u_{2}$ of Example 1 .

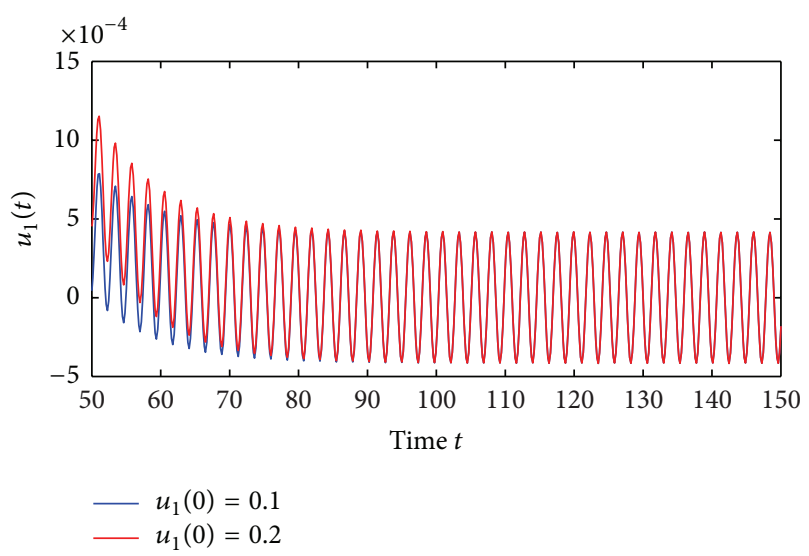

FIGURE 2: Exponential stability of state variable $u_{1}$ of Example 1.

It is easy to verify that all the conditions of Theorem 9 are satisfied and that the result follows from Theorem 9. Numerical simulations show that system (45) has a unique almost periodic solution, which is globally exponentially stable (see Figures 1, 2, and 3). This completes the proof.

In [28-30], the authors studied the existence and global exponential stability of almost periodic solution for a kind of Cohen-Grossberg neural networks without neutral delays based on the exponential dichotomy with fixed point theorem. Therefore, in some sense, we generalize the results in [28-30].

\section{Conclusions}

Compared with periodic effects, almost periodic effects are more frequent. In recent years, the study on the almost periodic behavior of neural networks has been the object of intensive analysis by numerous authors, and some of these results can be found in the literature. However, the neural networks of neutral type have not been fully investigated in the literature. In this paper, we give some sufficient conditions to ensure the existence, uniqueness, and global exponential stability of almost periodic solution for neutral-type CohenGrossberg neural networks. Numerical simulation is given to illustrate the feasibility of our main result. By using

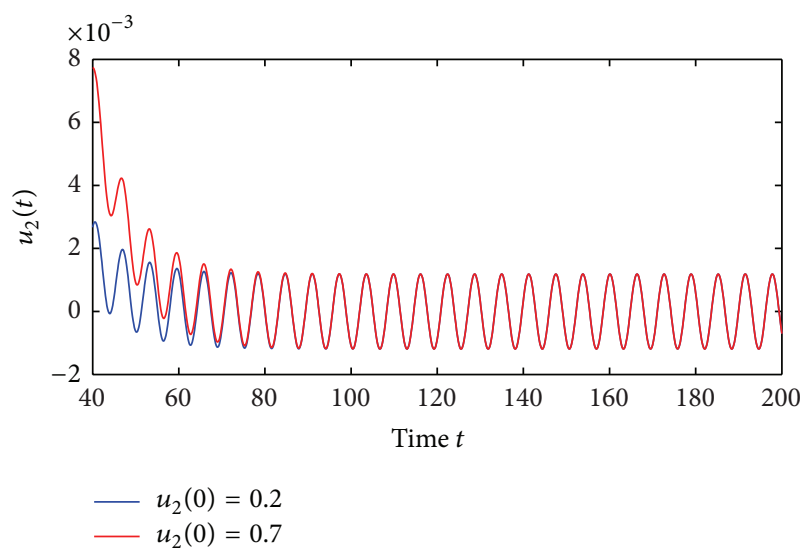

FIgURE 3: Exponential stability of state variable $u_{2}$ of Example 1 .

the method in this paper, we could study the existence, uniqueness, and global exponential stability of almost periodic solution of other (impulsive) neural networks (on time scales). We leave these for our future work.

\section{Acknowledgment}

The authors would like to thank the reviewers very much for their valuable suggestions on the paper.

\section{References}

[1] M. A. Cohen and S. Grossberg, "Absolute stability of global pattern formation and parallel memory storage by competitive neural networks," IEEE Transactions on Systems, Man, and Cybernetics, vol. 13, no. 5, pp. 815-826, 1983.

[2] Z. Zeng and J. Wang, "Global exponential stability of recurrent neural networks with time-varying delays in the presence of strong external stimuli," Neural Networks, vol. 19, no. 10, pp. 1528-1537, 2006.

[3] Z. Huang, S. Mohamad, and G. Cai, " $2^{N}$ almost periodic attractors for CNNs with variable and distributed delays," Journal of the Franklin Institute, vol. 346, no. 4, pp. 391-412, 2009.

[4] Z. Huang, S. Mohamad, X. Wang, and C. Feng, "Convergence analysis of general neural networks under almost periodic stimuli," International Journal of Circuit Theory and Applications, vol. 37, no. 6, pp. 723-750, 2009.

[5] C.-H. Li and S.-Y. Yang, "Existence and attractivity of periodic solutions to non-autonomous Cohen-Grossberg neural networks with time delays," Chaos, Solitons and Fractals, vol. 41, no. 3, pp. 1235-1244, 2009.

[6] Y. Li, "Existence and stability of periodic solutions for CohenGrossberg neural networks with multiple delays," Chaos, Solitons and Fractals, vol. 20, no. 3, pp. 459-466, 2004.

[7] C. Wu, J. Ruan, and W. Lin, "On the existence and stability of the periodic solution in the Cohen-Grossberg neural network with time delay and high-order terms," Applied Mathematics and Computation, vol. 177, no. 1, pp. 194-210, 2006.

[8] B. Liu and L. Huang, "Existence and exponential stability of periodic solutions for a class of Cohen-Grossberg neural networks with time-varying delays," Chaos, Solitons and Fractals, vol. 32, no. 2, pp. 617-627, 2007. 
[9] F. Long, Y. Wang, and S. Zhou, "Existence and exponential stability of periodic solutions for a class of Cohen-Grossberg neural networks with bounded and unbounded delays," Nonlinear Analysis: Real World Applications, vol. 8, no. 3, pp. 797-810, 2007.

[10] B. Li and D. Xu, "Existence and exponential stability of periodic solution for impulsive Cohen-Grossberg neural networks with time-varying delays," Applied Mathematics and Computation, vol. 219, no. 5, pp. 2506-2520, 2012.

[11] Y. Li, X. Chen, and L. Zhao, "Stability and existence of periodic solutions to delayed Cohen-Grossberg BAM neural networks with impulses on time scales," Neurocomputing, vol. 72, no. 7-9, pp. 1621-1630, 2009.

[12] X. Yang, "Existence and global exponential stability of periodic solution for Cohen-Grossberg shunting inhibitory cellular neural networks with delays and impulses," Neurocomputing, vol. 72, no. 10-12, pp. 2219-2226, 2009.

[13] Y. Li and X. Fan, "Existence and globally exponential stability of almost periodic solution for Cohen-Grossberg BAM neural networks with variable coefficients," Applied Mathematical Modelling, vol. 33, no. 4, pp. 2114-2120, 2009.

[14] Y. Li, T. Zhang, and Z. Xing, “The existence of nonzero almost periodic solution for Cohen-Grossberg neural networks with continuously distributed delays and impulses," Neurocomputing, vol. 73, no. 16-18, pp. 3105-3113, 2010.

[15] Q. Liu and R. Xu, "Periodic solutions of high-order CohenGrossberg neural networks with distributed delays," Communications in Nonlinear Science and Numerical Simulation, vol. 16, no. 7, pp. 2887-2893, 2011.

[16] Z. Zhang, G. Peng, and D. Zhou, "Periodic solution to CohenGrossberg BAM neural networks with delays on time scales," Journal of the Franklin Institute, vol. 348, no. 10, pp. 2759-2781, 2011.

[17] H. Xiang and J. Cao, "Exponential stability of periodic solution to Cohen-Grossberg-type BAM networks with time-varying delays," Neurocomputing, vol. 72, no. 7-9, pp. 1702-1711, 2009.

[18] H. Xiang, J. Wang, and J. Cao, "Almost periodic solution to Cohen-Grossberg-type BAM networks with distributed delays," Neurocomputing, vol. 72, no. 16-18, pp. 3751-3759, 2009.

[19] Y. Zhang, L. Guo, and C. Feng, "Stability analysis on a neutral neural network model," in Advances in Intelligent Computing, D.-S. Huang, X.-P. Zhang, and G.-B. Huang, Eds., vol. 3644 of Lecture Notes in Computer Science, pp. 697-706, 2005.

[20] S. Mandal and N. C. Majee, "Existence of periodic solutions for a class of Cohen-Grossberg type neural networks with neutral delays," Neurocomputing, vol. 74, no. 6, pp. 1000-1007, 2011.

[21] Z. Zhang, W. Liu, and D. Zhou, "Global asymptotic stability to a generalized Cohen-Grossberg BAM neural networks of neutral type delays," Neural Networks, vol. 25, pp. 94-105, 2012.

[22] V. Covachev, H. Akça, and M. Sarr, "Discrete-time counterparts of impulsive Cohen-Grossberg neural networks of neutral type," Neural, Parallel and Scientific Computations, vol. 19, no. 3-4, pp. 345-359, 2011.

[23] C. Bai, "Periodic oscillation for Cohen-Grossberg-type bidirectional associative memory neural networks with neutral time-varying delays," in Proceedings of the 5th International Conference on Natural Computation (ICNC '09), vol. 2, pp. 1823, Tianjin, China, August 2009.

[24] C.-D. Zheng, J.-W. Li, and Z. Wang, "New stability criteria for neutral-type Cohen-Grossberg neural networks with discrete and distributed delays," International Journal of Computer Mathematics, vol. 89, no. 4, pp. 443-466, 2012.
[25] A. M. Fink, Almost Periodic Differential Equations, vol. 377 of Lecture Notes in Mathematics, Springer, Berlin, Germany, 1974.

[26] M. Altman, "A fixed point theorem in Hilbert space," Bulletin of the Polish Academy of Sciences, vol. 5, pp. 19-22, 1957.

[27] D. J. Guo, Nonlinear Functional Analysis, Shandong Science and Technology Press, Shandong, China, 2003, (Chinese).

[28] H. Xiang and J. Cao, "Almost periodic solution of CohenGrossberg neural networks with bounded and unbounded delays," Nonlinear Analysis: Real World Applications, vol. 10, no. 4, pp. 2407-2419, 2009.

[29] H. Zhao, L. Chen, and Z. Mao, "Existence and stability of almost periodic solution for Cohen-Grossberg neural networks with variable coefficients," Nonlinear Analysis: Real World Applications, vol. 9, no. 2, pp. 663-673, 2008.

[30] Z. Chen, D. Zhao, and J. Ruan, "Almost periodic attractor for Cohen-Grossberg neural networks with delay," Physics Letters A, vol. 373, no. 4, pp. 434-440, 2009. 


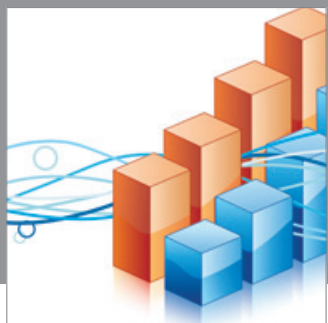

Advances in

Operations Research

mansans

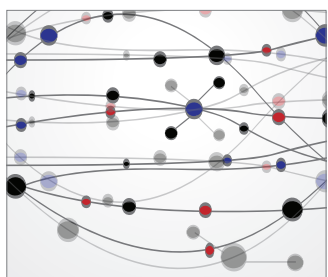

The Scientific World Journal
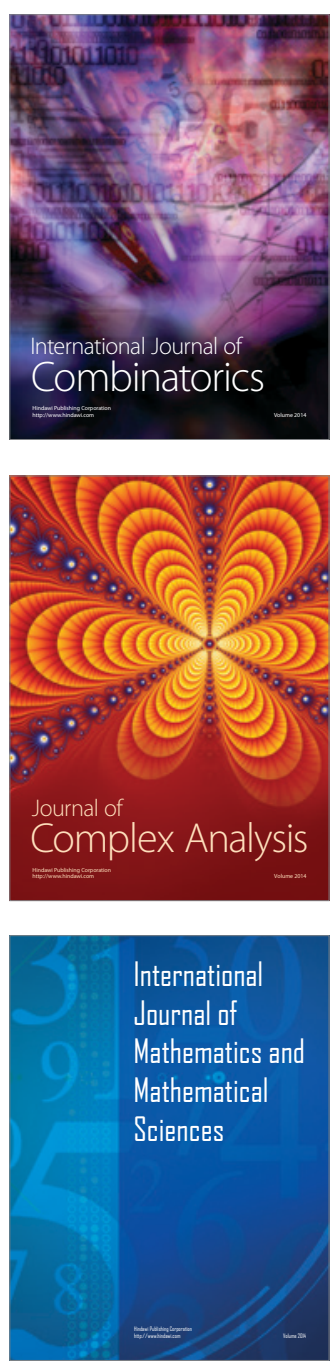
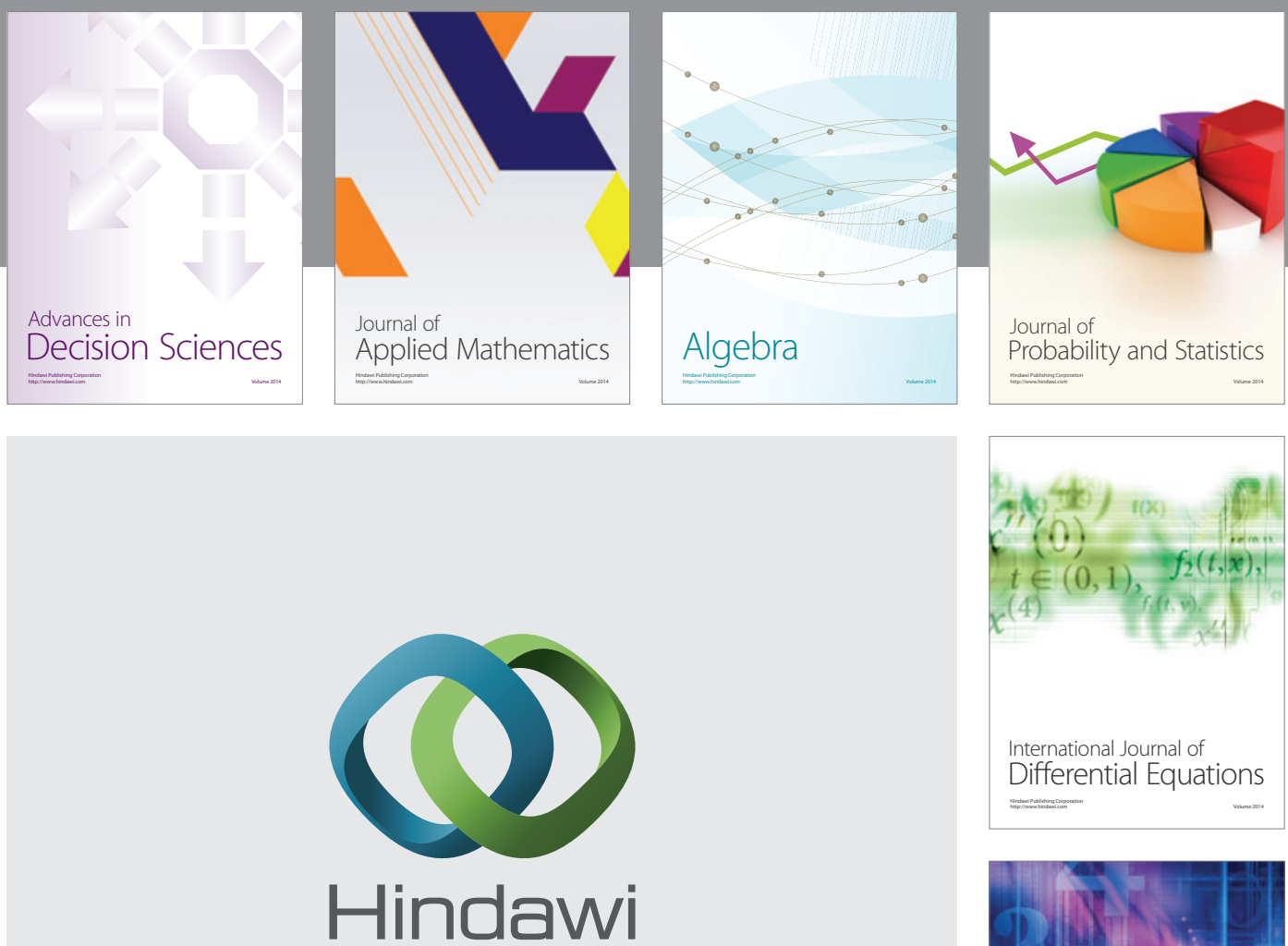

Submit your manuscripts at http://www.hindawi.com
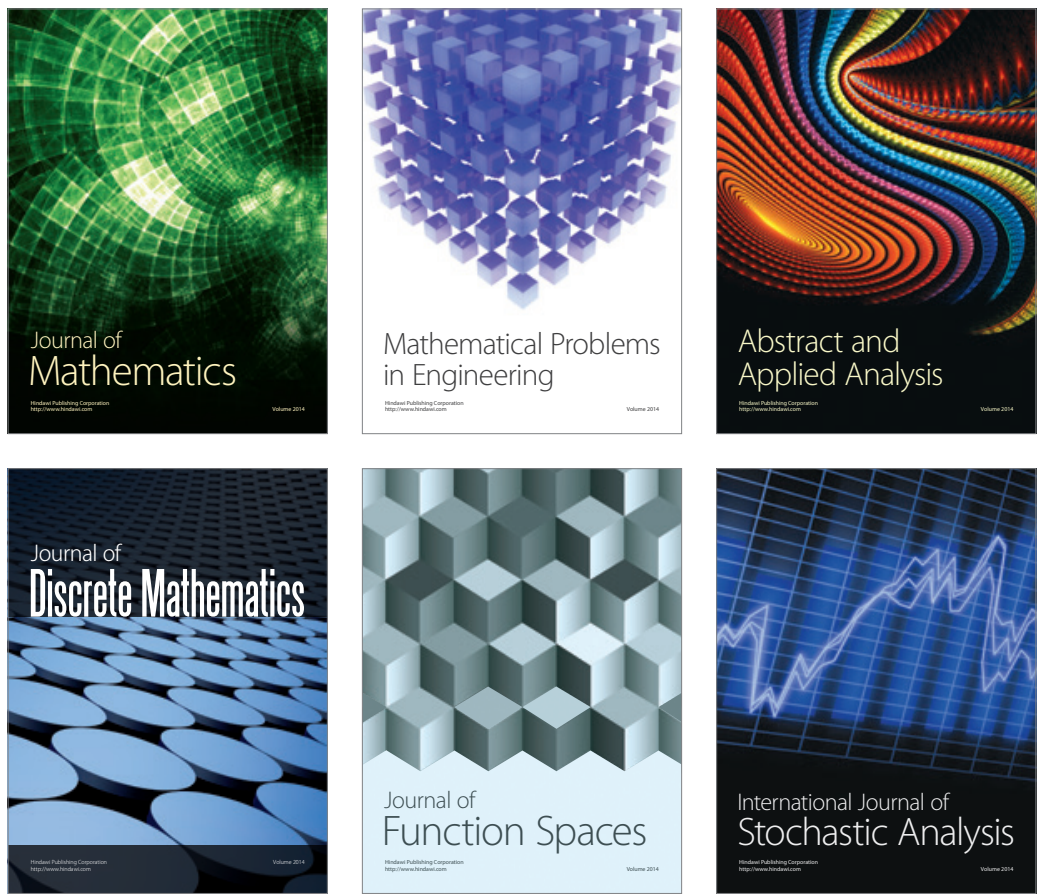

Journal of

Function Spaces

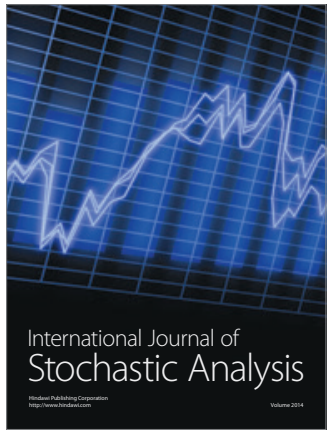

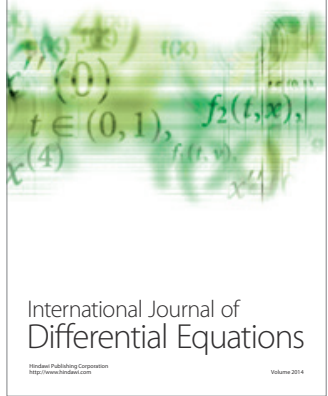
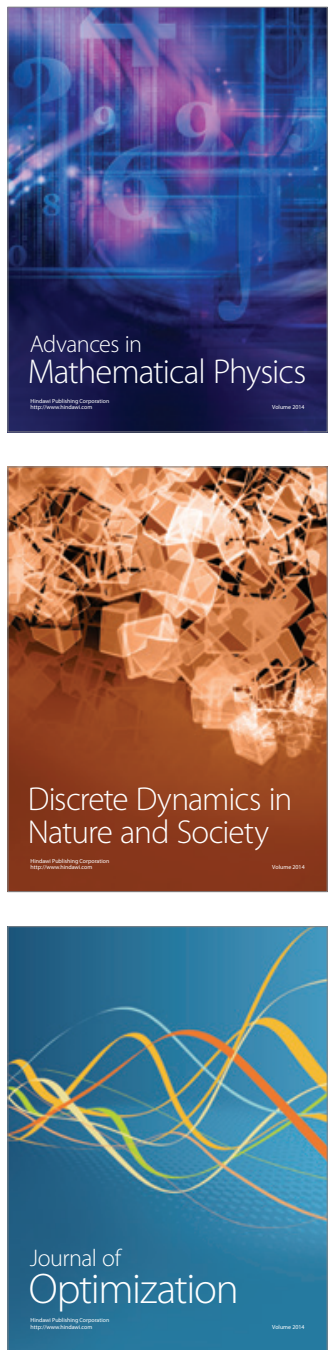